\title{
Different concepts of chronic musculoskeletal pain
}

The management of well defined musculoskeletal disorders with concordant symptoms and signs falls within the 'comfort zone' of most rheumatologists, yet much of one's time is spent outside this zone in dealing with patients whose reported pain experience is apparently discordant with discernible abnormalities. These are challenging patients. How one deals with them will depend on one's training, experience, inclination, and energy. Some will ultimately be referred to pain clinics, but high costs and limited access necessarily mean that a significant proportion continue to be managed by rheumatologists.

Somatic symptoms unaccompanied by detectable abnormalities may have two possible explanations: the first is a somatic disorder in which abnormalities are present but are so subtle so as to defy detection; the second is an emotional disorder of which the physical symptoms are a manifestation. It is in the latter cases that causal misattribution is common, and the doctor has a role in educating the patient. Some doctors find the first explanation the more appealing, either because intellectually they have some difficulty with the second, or simply because they wish to avoid a potentially acrimonious and embarrassing confrontation, knowing that even in an apparently 'enlightened' society most patients will reject any suggestion of abnormal or counterproductive cognition, with the perceived stigma of 'it's all in the mind'. ${ }^{1}$ Even while acknowledging the absence of supporting objective evidence, a doctor may be loth to discount an organic disorder, perhaps believing that medical science has not yet advanced sufficiently to provide the definitive diagnostic test. This is a facile argument which attempts to link a hypothetical as yet undiscovered test with a hypothetical medical disorder, suggesting that if the first were known the second would be proved, whereas in fact it is only from a knowledge of the pathophysiology that a confirmatory test can evolve, not the other way around.

\section{Non-disease and somatisation}

Not everyone is familiar with the concept of 'non-disease' described by Meador 30 years ago. ${ }^{2}$ Meador pointed out that patients are frequently referred with a specific disease diagnosis which subsequent investigation fails to substantiate. He suggested that, when a specific disease is suspected but not found, the patient has a particular nondisease. Rheumatologists not uncommonly encounter patients with upper limb paraesthesia in whom the clinical and electrodiagnostic tests are normal-perhaps an example of non-carpal tunnel syndrome?-or patients who anatomically localise pain to tendons about the wrists and distal forearms, but have none of the classical signs of inflammation - perhaps an example of non-tenosynovitis? Meador's paper was published 25 years before the term 'somatisation' found its place in the psychiatric lexicon. ${ }^{3}$ The term in part describes a patient's misattribution of bodily symptoms to organic disease. Once, these patients were pejoratively categorised as hysterics, hypochondriacs, or malingerers, but pioneers such as Pilowsky have provided alternative formulations of somatisation as learned or abnormal illness behaviour reinforced by the advantages of the sick role. ${ }^{4}$ Somatising patients do not lack psychological symptoms, but their preoccupation with their physical complaints means that they are less likely to report them and are more hostile to a diagnosis of mental illness. The problem is common. A British study of general practice found that $20 \%$ of patients present this way while, in contrast, only $5 \%$ of patients present with psychological complaints. ${ }^{5}$ The same study also found that some doctors might actually induce more somatisation in patients than others. Tyrer has pointed out that when expressions of pain produce sympathetic attention, the avoidance of unpleasant or stressful tasks, or are rewarded in more tangible ways, such behaviour is likely to be reinforced and will continue. This is referred to as learned pain behaviour. Its recognition 'depends on paying attention to the relation between pain behaviours and their apparent consequences-not in eliciting a multitude of inappropriate organic signs or symptoms'. ${ }^{6}$ Failure to recognise that pain is a symptom which does not necessarily indicate physical injury or disease could lead to misdiagnosis, particularly if the diagnosis is based on subjective rather than objective criteria. Somatising patients are all too receptive to diagnoses which carry the flavour of organicity and, from their viewpoint, authenticity ('now I know it's not in my head'). Musculoskeletal medicine provides several examples.

\section{'Soft tissue injury'}

It would be difficult to find a less helpful or more indeterminate 'diagnosis' than 'soft tissue injury' with no other qualification. Invariably, this describes a patient with a chronic pain complaint and tenderness on digital palpation in whom no discernible objective abnormality exists, for, were the case otherwise, a specific diagnosis would be made. Tenderness to palpation may be a useful localising sign in acute injury, but in patients with a chronic pain complaint, it is of very limited value. As Bogduk has pointed out with respect to notional soft tissue back injury, 'straining a muscle excessively results in damage to the myotendinous junction which attracts an inflammatory repair response ... this could be the sort of lesion that underlies an acute strained back but there is no evidence that sort of lesion can cause chronic low back pain ... muscle sprains of the back, like those of the limbs should heal and settle within a matter of days'.

\section{Myofascial pain syndrome (MPS)/fibromyalgia syndrome (FS)}

Despite a number of tantalising reports, ${ }^{8-10}$ the pathophysiology of MPS and FS remains indeterminate, and their diagnostic status syndromatic. The classically defined 'trigger point' ${ }^{11}$ once thought to distinguish MPS from FS is a relative rarity and cannot be used to distinguish the two disorders, while taut bands and muscle twitches are as common in healthy volunteers as in MPS and FS. ${ }^{12}$ Some authors have suggested that the 'tender points' of FS are an artefact of the physician's approach ${ }^{13}$ and even the experts acknowledge that examination technique may influence a patient's response. ${ }^{12}$ Does it matter? Perhaps acknowledging the 'legitimacy' of a patient's pain experience and addressing its underlying causes might ultimately prove to be of greater therapeutic value than labels which impute a knowledge of mechanisms and for which we have devised a range of empirical and often unhelpful treatments. 


\section{Unhelpful inferences}

The 'special test' has proved no less seductive than many of our diagnostic constructs and may be equally misleading, particularly if the pathology of the notional disorder is unknown or uncertain. The upper limb or brachial plexus tension test (BPTT) ${ }^{14}$ to diagnose repetitive strain injury (RSI) exemplifies this. Here, invention of a test has preceded discovery of pathophysiology, if indeed it exists. The BPTT is said to be analogous to the straight leg raise (SLR) test. True both are stretch tests, but that is where the similarity ends. The value of the SLR is that pain is provoked well within the normal range of elevation, there are often localising neurological signs, and the lumbar nerve root pathology which forms the rationale for the test is understood. In contrast, the BPTT induces pain at the extreme of the range, frequently requiring accessory stretch manoeuvres, is not accompanied by detectable neurological deficit, and is not based on any known pathology. Coincidentally, after the invention of the BPTT came the neurogenic hypothesis of RSI. ${ }^{15}$ Now, a notionally positive BPTT is 'evidence' of RSI and the neurogenic hypothesis, while the neurogenic hypothesis of RSI in turn is used to justify the use of the BPTT, one unknown effectively reinforcing another unknown. Curiously, although purportedly a neurological test, the BPTT has not gained favour amongst mainstream neurologists.

Wrist arthrography frequently detects anatomical aberrations, including carpal instability and breeches in the triangular fibrocartilage complex, yet, in a study of patients presenting with unilateral wrist pain in whom the asymptomatic wrist was used as the control, arthrographic abnormalities were to be found in the asymptomatic wrist in $74 \%$ of cases. ${ }^{16}$ The authors recommended that, because detectable changes were commonplace, bilateral arthrography in the investigation and management of unilateral wrist pain was mandatory.

What is true of wrist arthrography is equally true of other imaging techniques. For example, computed tomography and magnetic resonance imaging demonstrate herniated discs in more than $30 \%$ of individuals who have never had back pain. ${ }^{17}{ }^{18}$ As one group pointed out, 'the finding that an asymptomatic individual has more than a 1:4 chance of having an abnormal magnetic resonance image emphasises the danger of predicating a decision to operate on the basis of any diagnostic test in isolation without clinical information'. ${ }^{18}$
Although the practice of rheumatology has been greatly facilitated by a better understanding of biological mechanisms and by advances in medical technology, it is information gleaned from examination of the patient that remains the bedrock of clinical rheumatology. A normal examination is no less meaningful nor less rewarding than is the examination replete with abnormal findings; the interpretative processes no less complex. There is no necessity to compromise common sense for what is clinically untenable. If we are to serve our patients well, we need to consider not only what the clinical presentation may imply, but the reasons which might underlie it.

Pain Management Unit,

Stirling House, 28 Melbourne Street,

MARK AWERBUCH North Adelaide SA 5006, Australia

1 Hadler N M, ed. The dangers of the diagnostic process. Iatrogenic labelling as in the fibrositis paralogism. In: Occupational musculoskeletal disorders. New York: Raven Press, 1993; 16-33.

2 Meador C K. The art and science of nondisease. N Engl f Med 1965; 272: 92-5.

3 Murphy M. Somatisation: embodying the problem. BMF 1989; 298: 1331-2.

4 Pilowsky I. Abnormal illness behaviour. Br $\mathcal{f}$ Med Psychol 1969; 42: 347-51.

5 Goldberg D P, Bridges K. Somatic presentation of psychiatric illness in primary care settings. F Psychosom Res 1988; 32: 137-44.

6 Tyrer S P. Learned pain behaviour. BMf 1986; 292: 1

7 Bogduk N. The causes of low back pain. Med $\mathcal{f}$ Aust 1992; 156: 151-3.

8 Vaeroy H, Helle R, Forre O, Kass E, Terenius L. Elevated CSF levels of substance $P$ and high incidence of Raynaud's phenomenon in patients with fibromyalgia: new features for diagnosis. Pain 1988; 32: 21-6.

9 Bennett R M, Clark S R, Campbell S M, Burckhardt C S. Somatomedin $C$ levels in patients with the fibromyalgia syndrome: a possible link between sleep and muscle pain. Arthritis Rheum 1992; 35: 1113-6.

10 Yunus M B, Dailey J W, Aldag J C, Masi A T, Jobe P C. Plasma tryptophan and other amino acids in primary fibromyalgia: a controlled study. f Rheumatol 1992; 19: 90-4.

11 Travell J G, Simons D G. Myofascial pain and dysfunction: the trigger point manual. Baltimore: Williams and Wilkins, 1985.

12 Wolfe F, Simons D G, Friction J, et al. The fibromyalgia and myofascial pain syndromes: a preliminary study of tender points and trigger points in persons with fibromyalgia, myofascial pain syndrome and no disease. f Rheumatol 1992; 19: 944-51.

13 Quimby L G, Block S R, Gratwick M. Fibromyalgia: generalised pain intolerance and manifold symptom reporting. $\mathcal{F}$ Rheumatol 1988; 15: $1264-70$.

14 Elvey R L. Brachial plexus tension tests and the pathoanatomical origin of arm pain. In: Glasgow E F, Twomey L, eds. Aspects of manipulative therapy. Melbourne: Lincoln Institute of Health Sciences, $1979 ; 105-10$.

15 Cohen M L, Arroyo J F, Champion G D, Browne C D. In search of the pathogenesis of refractory cervicobrachial pain syndrome. A deconstruction of the RSI phenomenon. Med f Aust 1992; 156: 432-6.

16 Herbert J T, Faithfull R G, McCann D J, Ireland J. Bilateral arthrography of the wrist. 7 Hand Surg 1990; 15B: 233-5.

17 Wiesel S W, Tsoumaris N, Feffer H L, et al. A study of computer-assisted tomography. 1 . The incidence of positive CAT scans in an asymptomatic group of patients. Spine 1984; 9: 549-51.

18 Boden S D, Davis D O, Dina T S, et al. Abnormal magnetic-resonance scans of the lumbar spine in asymptomatic subjects. $\mathcal{F}$ Bone foint Surg Am 1990 72: 403-8. 